\title{
Centrally Reduced Diffusion Sign for Differentiation between Treatment-Related Lesions and Glioma Progression: A Validation Study
}

\author{
(D) P. Alcaide-Leon, (D). Cluceru, (D).M. Lupo, (D).J. Yu, (D)T.L. Luks, (DT. Tihan, (D) N.A. Bush, and (D).E. Villanueva-Meyer
}

\begin{abstract}
BACKGROUND AND PURPOSE: Differentiating between treatment-related lesions and tumor progression remains one of the greatest dilemmas in neuro-oncology. Diffusion MR imaging characteristics may provide useful information to help make this distinction. The aim of the study was to assess the diagnostic accuracy of the centrally reduced diffusion sign for differentiation of treatment-related lesions and true tumor progression in patients with suspected glioma recurrence.
\end{abstract}

MATERIALS AND METHODS: The images of 231 patients who underwent an operation for suspected glioma recurrence were reviewed. Patients with susceptibility artifacts or without central necrosis were excluded. The final diagnosis was established according to histopathology reports. Two neuroradiologists classified the diffusion patterns on preoperative MR imaging as the following: 1) reduced diffusion in the solid component only, 2) reduced diffusion mainly in the solid component, 3) no reduced diffusion, 4) reduced diffusion mainly in the central necrosis, and 5) reduced diffusion in the central necrosis only. Diagnostic accuracy metrics and the area under the receiver operating characteristic curve were estimated for the diffusion patterns.

RESULTS: One hundred three patients were included ( 22 with treatment-related lesions and 81 with tumor progression). The diagnostic accuracy results for the centrally reduced diffusion pattern as a predictor of treatment-related lesions ("mainly central" and "exclusively central" patterns versus all other patterns) were as follows: $64 \%$ sensitivity $(95 \% \mathrm{Cl}, 41 \%-83 \%), 84 \%$ specificity (95\% Cl, $74 \%-91 \%)$, 52\% positive predictive value $(95 \% \mathrm{Cl}, 37 \%-66 \%)$, and $89 \%$ negative predictive value $(95 \% \mathrm{Cl}, 83 \%-94 \%)$.

CONCLUSIONS: The centrally reduced diffusion sign is associated with the presence of treatment effect. The probability of a histologic diagnosis of a treatment-related lesion is low (11\%) in the absence of centrally reduced diffusion.

ABBREVIATION: ROC $=$ receiver operating characteristic

$\mathbf{T}$ he current standard-of-care treatment for newly diagnosed malignant gliomas is maximal safe resection followed by radiation with concurrent and adjuvant chemotherapy with temozolomide. ${ }^{1}$ These therapies often result in inflammation that can mimic tumor progression due to an increase in contrast enhancement on T1 postcontrast MR imaging. Recognizing treatment-related lesions is key for the adequate management of patients with glioma. Multiple advanced MR imaging techniques

Received December 19, 2019; accepted after revision June 29, 2020.

From the Department of Medical Imaging (P.A.-L.), University Health Network, Toronto, Ontario, Canada; and Departments of Radiology and Biomedical Imaging (J.C., J.M.L., T.J.Y., T.L.L., J.E.V.-M.), Pathology (T.T.), and Neurological Surgery (N.A. B.), University of California, San Francisco, San Francisco, California.

This work was supported by National Institutes of Health grants (P01 CA118816 and P50 CA097257).

Please address correspondence to Javier E. Villanueva-Meyer, Department of Radiology and Biomedical Imaging, UCSF, 505 Parnassus L-352, San Francisco, CA 94143; e-mail: javier.villanueva-meyer@ucsf.edu

- Indicates open access to non-subscribers at www.ajnr.org

http://dx.doi.org/10.3174/ajnr.A6843 including diffusion, perfusion, and MR spectroscopic imaging have been applied to solve this diagnostic challenge. ${ }^{2-8}$

Diffusion MR imaging is a technique that provides indirect information on the tissue microstructure through the quantification of the Brownian motion of water. The ADC is a measure of the magnitude of diffusion of water within tissue. Many studies have investigated the diagnostic value of the $\mathrm{ADC}$ for distinguishing recurrent glioma from treatment-related changes, but the findings have been incongruent, and $\mathrm{ADC}$ has been found to have moderate diagnostic performance. ${ }^{9}$ Both recurrent tumors and treatment-related lesions may have a heterogeneous appearance, with solid-enhancing and nonenhancing components as well as necrotic areas. Most studies have focused on the diffusion characteristics of the enhancing component, disregarding the necrotic areas because they were thought to contain no valuable information. However, a recent article by Zakhari et $\mathrm{al}^{10}$ describes the presence of centrally reduced diffusion in treatment-related necrosis in a small sample of patients. Quantification of ADC in the different lesion components provides 
objective measurements, but this approach is often not feasible because tumor segmentation remains time-consuming. In clinical practice, radiologists assess diffusion imaging patterns without ADC quantification. Therefore, a study focusing on imaging pattern more realistically estimates the practical diagnostic accuracy of the centrally reduced diffusion sign.

Our aim was to evaluate the diagnostic accuracy of the centrally reduced diffusion sign for differentiation between treatment-related lesions and true tumor progression in patients with suspected glioma recurrence.

\section{MATERIALS AND METHODS Subjects}

Institutional review board approval was obtained for this singlecenter retrospective study at the University of California San Francisco. We included patients with an initial pathologically confirmed diagnosis of World Health Organization grade II, III, or IV diffuse glioma treated with chemoradiation and a new or worsening enhancing lesion, undergoing surgery for suspected recurrence. Between October 2007 and November 2018, a total of 231 patients provided written informed consent to participate in a prospective radiologic-pathologic correlative study. A retrospective analysis was performed in this cohort. All patients underwent MR imaging between 1 and 3 days before surgical resection. Patients without macroscopic areas of necrosis were excluded. We considered necrosis a nonenhancing region with fluid signal intensity surrounded by contrast enhancement and showing a sum of biperpendicular diameters of $>10 \mathrm{~mm}$. We also excluded patients with susceptibility artifacts in the areas of necrosis to avoid the confounding effect of blood products on the diffusion evaluation. ${ }^{11}$

\section{MR Imaging Acquisition}

MR imaging examinations were performed on a $3 \mathrm{~T}$ scanner (Discovery 750, GE Healthcare, Waukesha, WI, USA) using an 8channel phased array head coil. For evaluation of contrast enhancement and central necrosis, we used a volumetric T1weighted inversion recovery spoiled gradient-echo sequence (TR/ $\mathrm{TE}=8.86 / 2.50 \mathrm{~ms}$, matrix $=256 \times 256$, section thickness $=$ $1.5 \mathrm{~mm}, \mathrm{FOV}=24 \times 24 \mathrm{~cm}, \mathrm{TI}=400 \mathrm{~ms}$, flip angle $\left.=15^{\circ}\right)$ before and after a $5-\mathrm{mL} / \mathrm{s}$ bolus injection of $0.1 \mathrm{mmol} / \mathrm{kg}$ body weight of gadopentetate dimeglumine (Magnevist; Bayer HealthCare Pharmaceuticals). For assessment of diffusion lesion characteristics, we used DWI with 6-directional axial diffusion EPI sequences $(\mathrm{TR} / \mathrm{TE}=7000-12,425 / 76-89 \mathrm{~ms}$, matrix $=256 \times$ $256 \times 120$, section thickness $=1.5 \mathrm{~mm}, \mathrm{FOV}=24 \times 24 \times$ $18 \mathrm{~cm}, b=1000 \mathrm{~s} / \mathrm{mm}^{2}$, number of excitations = 4) or DWI with 3-directional axial EPI sequences (TR/TE $=13,800 /$ $80.2 \mathrm{~ms}$, matrix $=110 \times 116$, section thickness $=2.5 \mathrm{~mm}$, $\mathrm{FOV}=25 \times 22.5 \mathrm{~cm}, b=1000 \mathrm{~s} / \mathrm{mm}^{2}$, number of excitations $=$ 4). ADC maps were generated from diffusion images. The presence of susceptibility artifacts was assessed on the $\mathrm{T} 2{ }^{*}$ weighted angiography sequence $(\mathrm{TE}=26 \mathrm{~ms}, \mathrm{TR}=50 \mathrm{~ms}$, $\mathrm{FOV}=256 \times 256 \mathrm{~cm}$, matrix size $=300 \times 300$, resolution $=$ $0.9375 \times 0.9375 \mathrm{~mm})$. In the absence of a T2*-weighted angiography sequence, a T2*-weighted EPI sequence was used $\left(\mathrm{TE} /\right.$ flip angle $=25-45 \mathrm{~ms} / 35^{\circ}$, matrix $=256 \times 256$, section thickness $=5 \mathrm{~mm}$ ).

\section{Image Interpretation}

Screening of patients for inclusion in the study was performed by a neuroradiologist with 9 years of experience (P.A.-L). Patients lacking presurgical DWI, lacking an area of necrosis, or presenting with susceptibility artifacts due to blood products were excluded. Twenty percent of the potentially eligible patients $(n=49)$ were also assessed by a different neuroradiologist (J.E.V.-M.) for the assessment of interobserver agreement. Disagreements were resolved by consensus. Two neuroradiologists (P.A.-L. and J.E.V.-M.), independently and blinded to the pathology report, evaluated the diffusion images of all patients included in the study. Disagreements were resolved by consensus. We evaluated diffusion characteristics of 2 distinct components of new or worsening lesions, the solid portion, comprising enhancing and nonenhancing signal abnormality, and the central necrotic portion. Diffusion images including $b=1000$ trace images and ADC maps were interpreted in conjunction with postcontrast $\mathrm{T} 1$ images to classify the lesions according to 5 diffusion patterns. We considered areas of reduced diffusion those regions showing hyperintensity on $b=1000$ trace images with corresponding $\mathrm{ADC}$ values lower or equal to those of normal-appearing white matter. On the basis of the results of prior studies, ${ }^{10,12}$ the diffusion patterns were assigned an ordinal scale with a higher score representing a higher probability of a treatment-related lesion and a lower probability of a recurrent tumor. Diffusion patterns were categorized as follows: 1, reduced diffusion in the solid component only; 2, reduced diffusion mainly in the solid component; 3 , no reduced diffusion; 4 , reduced diffusion mainly in the central necrosis; and 5, reduced diffusion in the central necrosis only.

\section{Histopathology Interpretation}

The final diagnosis of a treatment-related lesion or recurrent tumor was assigned on the basis of the pathology report extracted from the electronic medical records. At our institution, pathology in each glioma case is reviewed by an experienced neuropathologist. Cases were assigned to the treatment-related lesion category when treatment-related changes were present and $<25 \%$ of viable tumor was identified. Cases with $\geq 25 \%$ viable tumor were classified as recurrent tumor regardless of the presence of treatment effect. This threshold was used in a prior study ${ }^{13}$ and is commonly used in clinical decision-making at our institution. When described on the pathology report, percentages of treatment effect and viable tumor were recorded. The histology of cases classified as tumor $(>25 \%$ of viable tumor) with available slides was re-reviewed by an expert neuropathologist (T.T.) to investigate the impact of mixed lesions in the diagnostic accuracy of the centrally reduced diffusion sign.

\section{Statistical Analysis}

The Cohen $\kappa^{14}$ was calculated to identify the interobserver agreement for the presence of central necrosis and blood. The linearly weighted Cohen $\kappa^{15}$ was calculated to identify the interobserver agreement for the presence of the different diffusion patterns. A $\kappa$ value of $\leq 0.2$ indicates slight agreement; $0.21-0.4$, fair agreement; $0.41-0.6$ moderate agreement; $0.61-0.80$, substantial agreement, and 0.81-1.00, almost perfect agreement. ${ }^{16}$

Predictive performance of the diffusion patterns was assessed by estimating negative and positive predictive values, sensitivity and specificity, and the area under the receiver operating characteristic 
(ROC) curve, with corresponding 95\% confidence intervals. We also compared the diagnostic accuracy of the traditional diffusion approach that only considers the diffusion values in the solid regions and the diffusion assessment using the centrally reduced diffusion sign. The McNemar test was used for diagnostic accuracy comparison.

We also investigated the presence of mixed lesions as a cause of false-positive results of the centrally reduced diffusion sign. Within the tumor group ( $>25 \%$ of recurrent tumor), we compared the percentages of treatment effect between patients with predominant centrally reduced diffusion and patients with other diffusion patterns using the Mann-Whitney $U$ test. Statistical analyses were performed using GraphPad Prism software, Version 8.2.1 (GraphPad Software).

\section{RESULTS}

\section{Baseline Characteristics}

A total of 231 patients with glioma with a new-or-worsening enhancing lesion undergoing an operation at our institution were enrolled between October 2007 and November 2018. Patients

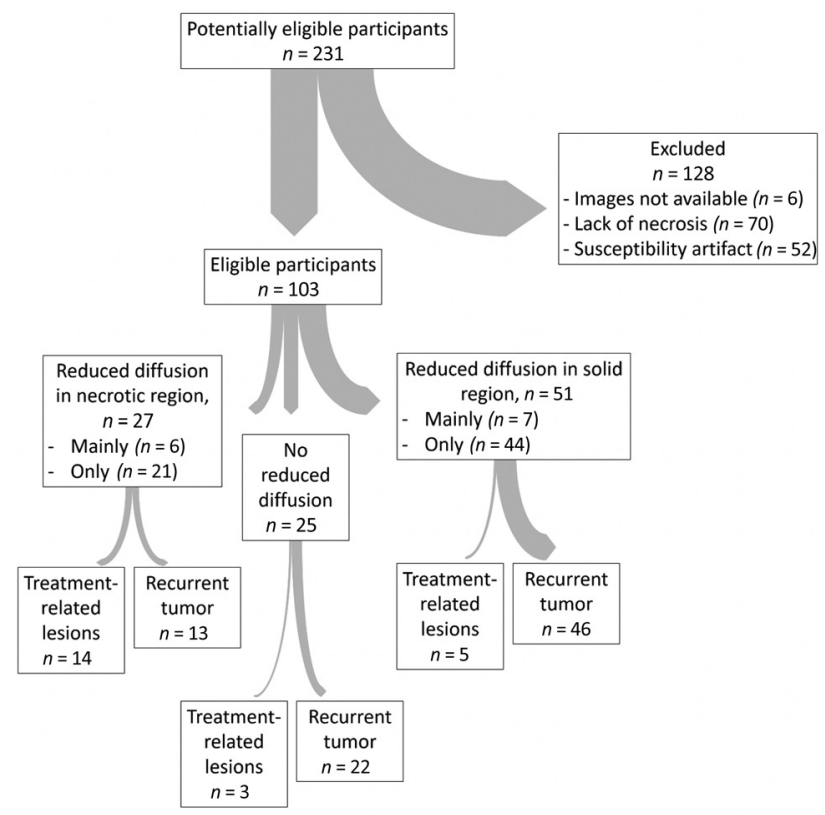

FIG 1. Standards for the Reporting of Diagnostic Accuracy Studies flow diagram. The thickness of the arrows is proportional to the number of subjects. without areas of visible necrosis $(n=70)$, with susceptibility artifacts $(n=52)$, or without available images $(n=6)$ were excluded. A total of 103 eligible patients (44 women and 59 men) were included for analysis, 81 (79\%) with recurrent tumor and 22 (21\%) with treatment-related lesions. The mean age at surgery for suspected recurrence was 53 years (range, 25-84 years). The Standards for the Reporting of Diagnostic Accuracy Studies (https://bmjopen.bmj.com/content/6/11/e012799) flow diagram is shown in Fig 1. Stereotactic biopsy was performed in 1 patient; gross total resection, in 42 patients; and subtotal resection, in 60 patients. The original tumor histology was oligodendroglioma grade II in 3 cases, oligodendroglioma grade III in 2 cases, oligoastrocytoma grade II in 1 case, oligoastrocytoma grade III in 2 cases, astrocytoma grade II in 1 case, astrocytoma grade III in 13 cases, and glioblastoma/gliosarcoma in 81 cases. Most cases were diagnosed before 2016; therefore, a mix of the World Health Organization classifications of 2007 and 2016 was used. The date of the end of radiation treatment was available in 75/103 patients. The median time from the end of radiation treatment to the preoperative MR imaging was 7.8 months for the treatment-effect group (95\% CI, 3.8-14 months) and 17 months for the recurrenttumor group (95\% CI, 9.4-29 months).

\section{Interobserver Agreement}

The $\kappa$ value was 0.7 (95\% CI, 0.5-0.9) for the presence of central necrosis and 0.8 (95\% CI, 0.7-1) for the presence of blood products. The linearly weighted $\kappa$ value for the diffusion patterns was 0.7 (95\% CI, 0.6-0.8).

\section{Predictive Value of the Diffusion Patterns}

The number of patients showing each diffusion pattern in the recurrent tumor group and in the treatment-related lesions group is shown in the Table and displayed graphically in Fig 2. Figure 3 shows typical diffusion patterns of treatment-related lesions and recurrent tumor.

The diagnostic-accuracy results for the centrally reduced diffusion pattern as a predictor of treatment-related lesions (mainly central and exclusively central patterns versus all other patterns) were as follows: $80 \%$ accuracy (95\% CI, 71\%-87\%), 64\% sensitivity (95\% CI, 41\%-83\%), 84\% specificity (95\% CI, 74\%-91\%), $52 \%$ positive predictive value $(95 \% \mathrm{CI}, 37 \%-66 \%)$, and $89 \%$ negative predictive value (95\% CI, 83\%-94\%). The diagnostic accuracy results for the traditional approach that considers only the presence of reduced diffusion in the solid lesion component as a

Distribution of the different diffusion patterns in the treatment-related lesion and recurrent-tumor groups

\begin{tabular}{lcccc}
\multicolumn{1}{c}{ Diffusion Pattern } & $\begin{array}{c}\text { Treatment- } \\
\text { Related Lesions }\end{array}$ & $\begin{array}{c}\text { Percentage of Total Cases of } \\
\text { Treatment-Related Lesions }\end{array}$ & $\begin{array}{c}\text { Recurrent } \\
\text { Tumor }\end{array}$ & $\begin{array}{c}\text { Percentage of Total Cases of } \\
\text { Recurrent Tumor }\end{array}$ \\
\hline $\begin{array}{l}\text { Reduced diffusion in the solid } \\
\text { region }\end{array}$ & 3 & $13.64 \%$ & 41 & $50.62 \%$ \\
$\begin{array}{l}\text { Reduced diffusion mainly in the } \\
\text { solid region }\end{array}$ & 2 & $9.1 \%$ & 5 & $6.17 \%$ \\
$\begin{array}{l}\text { No reduced diffusion } \\
\begin{array}{l}\text { Reduced diffusion mainly in the } \\
\quad \text { necrotic region }\end{array}\end{array}$ & 3 & $13.64 \%$ & 22 & $27.16 \%$ \\
$\begin{array}{l}\text { Reduced diffusion in the } \\
\text { necrotic region }\end{array}$ & 11 & $13.64 \%$ & 3 & $3.70 \%$ \\
All & 22 & $50 \%$ & 10 & $12.35 \%$ \\
\hline
\end{tabular}




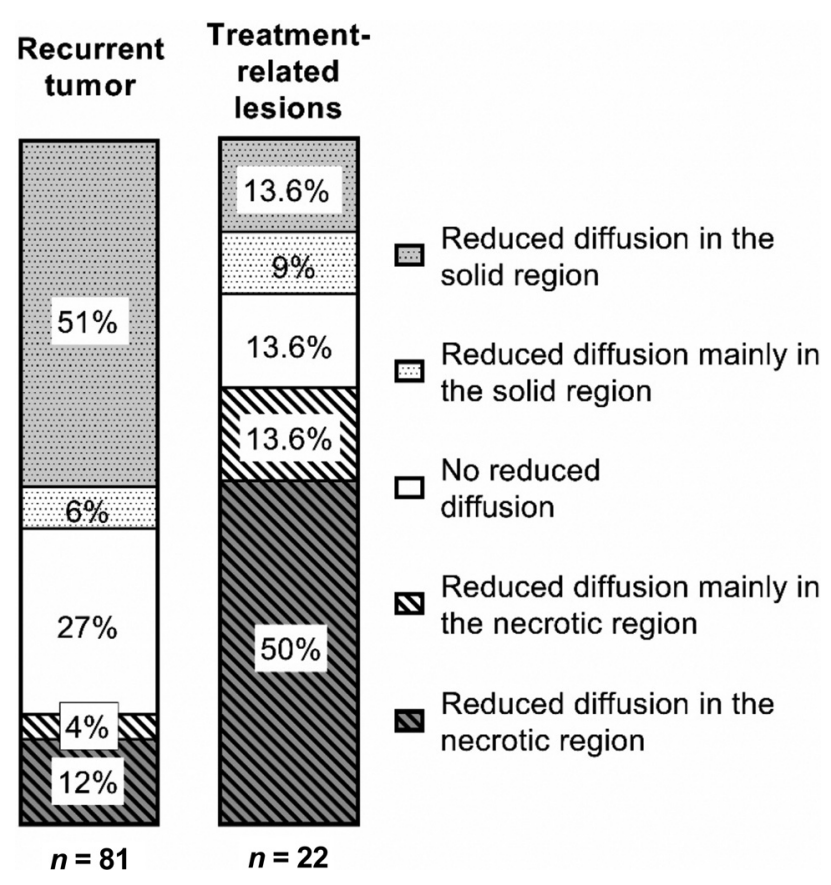

FIG 2. Distribution of the different diffusion patterns in the treatment-related lesion and recurrent-tumor groups.

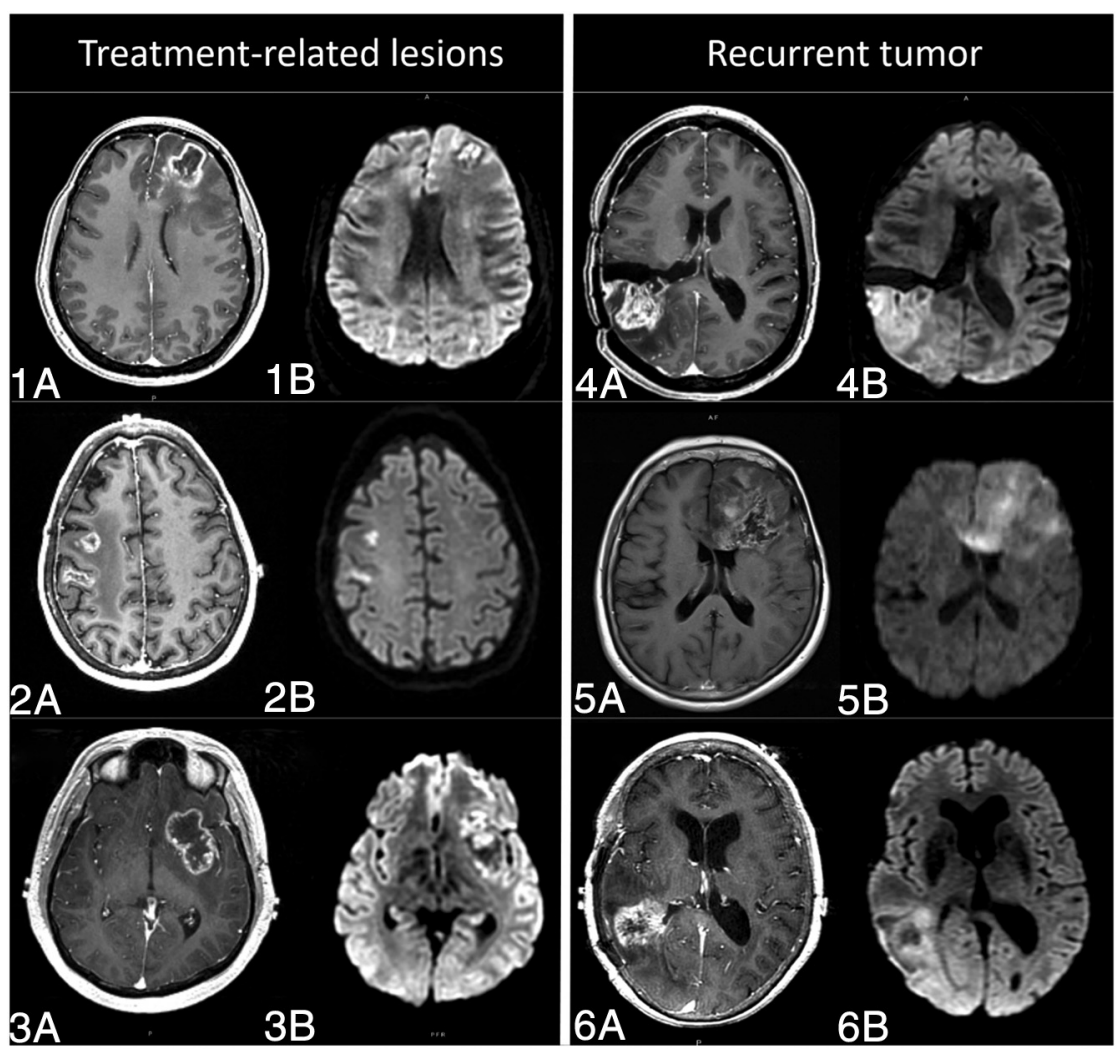

FIG 3. Preoperative MR imaging of 6 patients with suspected glioma recurrence. In 3 cases (1, 2 , and 3), histopathology revealed treatment-related lesions, and in the other 3 cases (4, 5, and 6), recurrent tumor. T1-weighted postcontrast $(A)$ and DWI $(B)$ are shown in each case. Treatmentrelated lesions (1, 2, and 3) show reduced diffusion predominantly in the central necrotic region. Lesions corresponding to progressive tumor (4, 5, and 6), on the other side, show reduced diffusion mainly in the solid-lesion components. marker of recurrent tumor (mainly solid and exclusively solid diffusion patterns versus all other patterns) were as follows: $61 \%$ accuracy (95\% CI, 51\%-71\%), 57\% sensitivity (95\% CI, 45\%-68\%), $77 \%$ specificity (95\% CI, 55\%-92\%), 90\% positive predictive value (95\% CI, 81\%-95\%), and 33\% negative predictive value (95\% CI, 26\%-40\%). The diagnostic accuracy of the centrally reduced diffusion sign was significantly higher than that of the traditional diffusion-assessment approach that considers only the reduced diffusion of the solid lesion components $(P<0.001)$.

\section{ROC Analysis of Diffusion Patterns}

The ROC curve for the diffusion patterns as a predictor of treatment-related lesions (positive state) or recurrent tumor (negative state) is shown in Fig 4. The area under the ROC curve was 0.77 (95\% CI, 0.7-0.9). The best cutoff point for predicting the treatment effect was $\geq 4$. In the ordinal scale assigned to the diffusion patterns, 4 corresponded to reduced diffusion located, mainly but not exclusively, in the necrotic region, whereas 5 corresponded to reduced diffusion present exclusively in the necrotic region.

\section{Subgroup Analysis of False-Positive Cases}

Among the patients with recurrent tumor $(n=81)$, the percentage of treatment effect in tissue samples was obtained by pathology re-evaluation for 33 patients with available and sufficient slides. Of the 48 patients without available or sufficient slides, we were able to find the percentage of treatment effect in the clinical pathology report for 7 additional patients. Percentages of treatment effect were available in a total of 40 patients classified as having recurrent tumor $(>25 \%$ of viable tumor), combining the reevaluation of slides and the clinical pathology report. Eleven cases showed a predominant pattern of centrally reduced diffusion, and 29 showed other patterns. In 2 cases, pathologic rereview described $>75 \%$ of the treatment effect in cases originally classified as recurrent tumor according to initial clinical pathology. In these 2 cases, we decided to keep the clinical diagnosis of recurrent tumor because we cannot confirm that all original slides were available for re-evaluation. The median of the percentage of concurrent treatment effect in patients with tumor recurrence was 30\% (95\% CI, 20\%-45\%) in patients with a pattern of centrally reduced diffusion and 40\% (95\% CI, $30 \%-75 \%$ ) in patients with other diffusion patterns. The differences were not statistically significant (Mann-Whitney $U$ test, $P$ value $=.076)$. Figure 5 shows the distribution of the percentages of concurrent treatment effect by diffusion pattern. 


\section{ROC curve for diffusion patterns}

(treatment-related lesion=positive state)
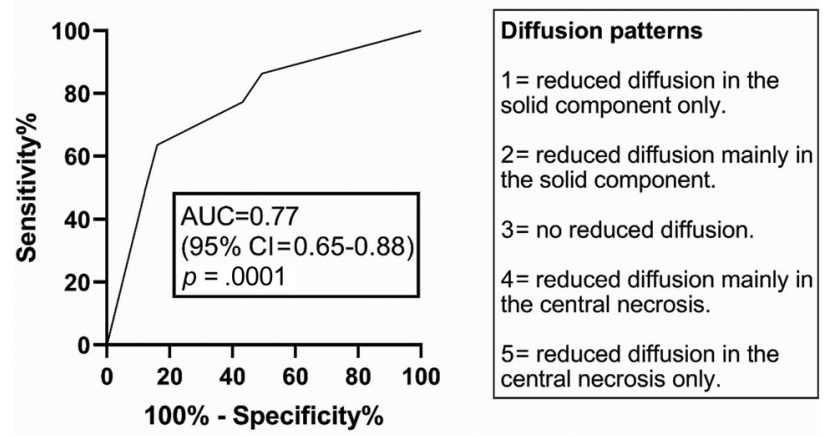

FIG 4. ROC curve for diffusion patterns. AUC indicates area under the curve.

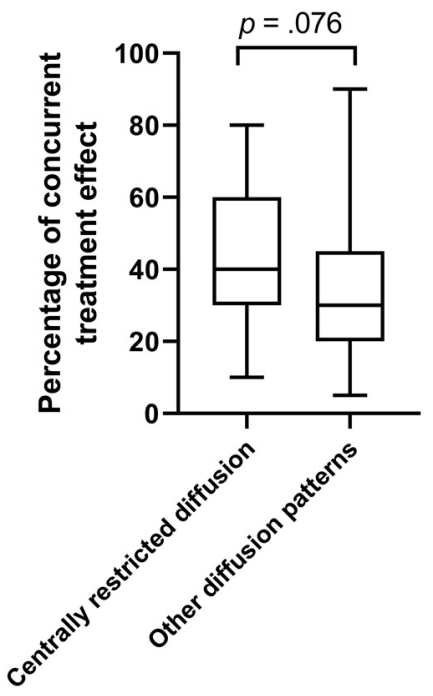

FIG 5. The box-and-whisker plot shows the distribution of the percentages of concurrent treatment effect in patients with recurrent tumor by diffusion pattern. The whiskers mark the maximum and minimum values, and the box edges indicate the first and third quartiles. The horizontal line inside the box corresponds to the median. The median percentage of treatment effect was higher in the group with centrally reduced diffusion than in the group with the other diffusion patterns, but differences were not statistically significant $(P=.076)$.

\section{DISCUSSION}

The present study found an overall diagnostic accuracy of $80 \%$ for the centrally reduced diffusion sign in the differentiation between treatment effect and glioma recurrence. The negative predictive value for a predominantly central reduced diffusion pattern was $89 \%$. The absence of a predominant pattern of centrally reduced diffusion in a ring-enhancing lesion suspicious for glioma recurrence implies only around an $11 \%$ chance that the lesion corresponds to treatment effect. When a predominant pattern of centrally reduced diffusion was present, the probability of a lesion being a treatment effect was $51 \%$ (positive predictive value). We believe the low positive predictive value of the centrally reduced diffusion sign is likely related to the presence of mixed lesions. In the present study, mixed lesions were more likely to be histologically classified as recurrent tumor because a mixed lesion had to show only $>25 \%$ of viable tumor tissue to be classified as recurrent tumor. We hypothesized that a number of mixed lesions with extensive treatment effect and centrally reduced diffusion were classified as recurrent tumor and therefore deemed false-positives. To evaluate this hypothesis, we compared the percentages of concurrent treatment effect in cases classified as recurrent tumor with and without a predominant pattern of centrally reduced diffusion. Unfortunately, the percentage of treatment effect was only available in 40 cases of recurrent tumor, and though the median of the percentages of treatment effect was higher in the patients with recurrent tumor with centrally reduced diffusion than without it (40\% versus $30 \%$ ), the differences were not statistically significant. The diagnostic accuracy of the centrally reduced diffusion sign was significantly higher than that of the traditional diffusion assessment that considers only the solid lesion components. Our results suggest that diffusion restriction in the solid component is a marker of recurrent tumor, whereas the reduced diffusion in the necrotic region is associated with treatment effect. Given the high prevalence of mixed lesions in the context of suspected glioma recurrence, we find the complementary information provided by the diffusion in the solid and necrotic lesion components extremely valuable.

Both the imaging exclusion criteria (lack of necrotic areas and presence of susceptibility) and the diagnostic test (diffusion patterns) showed substantial interobserver reliability $(\kappa=0.7-0.8)$. In the prior study ${ }^{10}$ describing the centrally reduced diffusion sign, a single neuroradiologist assessed the images; therefore, interobserver reliability was not evaluated. For a diagnostic sign to be valuable, it must be shown to be reliable when used by different practitioners. Our work not only validates the findings of the prior study but also adds the interobserver reliability assessment.

Diffusion MR imaging has been widely studied for differentiation of recurrent tumor and treatment-related lesions, but prior studies show inconsistent results. ${ }^{6,17}$ One of the reasons for the inconsistency may be methodologic because prior studies differ in the location and method of diffusion measurements. To account for lesion heterogeneity, some studies selected portions of the ADC histogram, either with formal histogram analysis or by manually selecting ROIs with the lowest or highest $\mathrm{ADC}$ values. In some studies, the smallest $\mathrm{ADC}$ values within the enhancing lesion were the most useful, ${ }^{7}$ whereas others assigned the best discriminative power to the maximum $\mathrm{ADC}$ values. ${ }^{3}$ Most studies focused on the evaluation of the enhancing lesion component exclusively, ${ }^{3,5}$ whereas other studies evaluated the whole lesion. ${ }^{7}$ Inconsistent and even contradictory results of prior studies are not surprising because they do not account for the fact that the central coagulative necrosis in treatment-related lesions can restrict water motion as much as tumor cellularity can. ${ }^{18}$

A study using a delayed radiation necrosis model in rats showed significantly lower ADC in the necrotic central zone than in the peripheral zone. Histologic analysis revealed parenchymal coagulative necrosis in the central zone and damaged vessels and reactive astrogliosis in the peripheral zone. ${ }^{18}$ Zakhari et $\mathrm{al}^{10}$ described the presence of centrally reduced diffusion in treatment-related necrosis and the diagnostic accuracy of the central/peripheral ADC ratio for differentiation of radiation necrosis and recurrent glioma. They evaluated a sample of 17 patients including 9 cases of recurrent tumor and 8 cases of radiation necrosis. We believe the proportion of radiation necrosis found in this study was very high, despite it being a prospective study with consecutive patient enrollment; this feature should capture the real prevalence of radiation necrosis and 
recurrent tumor. The prevalence of treatment-related lesions in our cohort was $21 \%$. The reason for this mismatch may be the small sample of the prior study and the fact that they excluded patients with equal amounts of treatment effect and recurrent tumor. Using our criteria, those patients would have been classified as having recurrent tumor. The present study evaluates not only a larger but also a more representative sample of patients undergoing an operation for suspected glioma recurrence, which allows positive and negative predictive value estimation. Our results consolidate the presence of centrally reduced diffusion as highly specific for detection of treatment-related lesions in the context of a new or worsening ring-enhancing lesion suspicious for glioma recurrence.

\section{Limitations}

The study has some limitations. First, the presence of a concurrent treatment effect was not available in a large portion of patients, precluding a meaningful subanalysis of the false-positive cases. Second, we performed only qualitative evaluation of the diffusion patterns, and we made no distinction among different fractions of reduced diffusion in the solid and necrotic components. We think this is a more broadly applicable approach that better recreates the interpreting physician's clinical practice at the expense of less objective information. Third, the prevalence of treatment-related lesions and, therefore, the predictive values were estimated in a cohort of patients undergoing an operation for suspected glioma recurrence. This assumption may carry an institutional bias because surgical practice patterns in this setting may differ across institutions. Fourth, a few patients with an original diagnosis of low-grade glioma were included in the study together with a majority of patients with higher-grade gliomas. Recurrence in high and low grades may manifest differently, but the low number of patients with low grade gliomas precluded subgroup analysis. Fifth, the radiation dose was not available in all patients; this feature may influence the pretest probability of radionecrosis. Sixth, information on whether treatment effect or tumor recurrence was suspected in each case before the operation was not available for all patients.

\section{CONCLUSIONS}

The centrally reduced diffusion sign is highly specific for detecting treatment-related lesions in the context of suspected glioma recurrence. Our findings contradict the traditional assumption that central necrosis contains no valuable information for differentiation of treatment effect and recurrent tumor. Further studies intending to use diffusion or other imaging techniques for differentiating treatment effect and recurrent tumor should also take into account the information extracted from the necrotic portions of the lesions.

\section{ACKNOWLEDGMENTS}

The authors wish to acknowledge the generous support of the Slaight Family Foundation.

Disclosures: Paula Alcaide-Leon—RELATED: Grant: National Institutes of Health.* Janine M. Lupo—RELATED: Grant: National Institutes of Health*; Support for Travel to Meetings for the Study or Other Purposes: National Institutes of Health*; UNRELATED: Grants/Grants Pending: National Institutes of Health, GE Healthcare*; Travel/Accommodations/Meeting Expenses Unrelated to Activities
Listed: National Institutes of Health.* Javier E. Villanueva-Meyer-UNRELATED: Grants/Grants Pending: GE Healthcare.* *Money paid to the institution.

\section{REFERENCES}

1. Stupp R, Mason WP, van den Bent MJ, et al. Radiotherapy plus concomitant and adjuvant temozolomide for glioblastoma. $\mathrm{N} \mathrm{Engl} \mathrm{J}$ Med 2005;352:987-96 CrossRef Medline

2. Alexiou GA, Zikou A, Tsiouris S, et al. Comparison of diffusion tensor, dynamic susceptibility contrast MRI and $99 \mathrm{~m}$ Tc-Tetrofosmin brain SPECT for the detection of recurrent high-grade glioma. Magn Reson Imaging 2014;32:854-59 CrossRef Medline

3. Asao C, Korogi Y, Kitajima M, et al. Diffusion-weighted imaging of radiation-induced brain injury for differentiation from tumor recurrence. AJNR Am J Neuroradiol 2005;26:1455-60 Medline

4. Ceschin R, Kurland BF, Abberbock SR, et al. Parametric response mapping of apparent diffusion coefficient as an imaging biomarker to distinguish pseudoprogression from true tumor progression in peptide-based vaccine therapy for pediatric diffuse intrinsic pontine glioma. AJNR Am J Neuroradiol 2015;36:2170-76 CrossRef Medline

5. Hein PA, Eskey CJ, Dunn JF, et al. Diffusion-weighted imaging in the follow-up of treated high-grade gliomas: tumor recurrence versus radiation injury. AJNR Am J Neuroradiol 2004;25:201-09 Medline

6. Lee WJ, Choi SH, Park CK, et al. Diffusion-weighted MR imaging for the differentiation of true progression from pseudoprogression following concomitant radiotherapy with temozolomide in patients with newly diagnosed high-grade gliomas. Acad Radiol 2012;19:135361 CrossRef Medline

7. Matsusue E, Fink JR, Rockhill JK, et al. Distinction between glioma progression and post-radiation change by combined physiologic MR imaging. Neuroradiology 2010;52:297-306 CrossRef Medline

8. Prager AJ, Martinez N, Beal K, et al. Diffusion and perfusion MRI to differentiate treatment-related changes including pseudoprogression from recurrent tumors in high-grade gliomas with histopathologic evidence. AJNR Am J Neuroradiol 2015;36:877-85 CrossRef Medline

9. Zhang H, Ma L, Shu C, et al. Diagnostic accuracy of diffusion MRI with quantitative $\mathrm{ADC}$ measurements in differentiating glioma recurrence from radiation necrosis. J Neurol Sci 2015;351:65-71 CrossRef Medline

10. Zakhari N, Taccone MS, Torres C, et al. Diagnostic accuracy of centrally restricted diffusion in the differentiation of treatmentrelated necrosis from tumor recurrence in high-grade gliomas. AJNR Am J Neuroradiol 2018;39:260-64 CrossRef Medline

11. Atlas SW, Dubois P, Singer MB, et al. Diffusion measurements in intracranial hematomas: Implications for MR imaging of acute stroke. AJNR Am J Neuroradiol 2000;21:1190-94 Medline

12. Alcaide-Leon P, Luks TL, Lafontaine M, et al. Treatment-induced lesions in newly diagnosed glioblastoma patients undergoing chemoradiotherapy and heat-shock protein vaccine therapy. J Neurooncol 2020;146:71-73 CrossRef Medline

13. Wang S, Sakai Y, Chawla S, et al. Differentiating tumor progression from pseudoprogression in patients with glioblastomas using diffusion tensor imaging and dynamic susceptibility contrast MRI. AJNR Am J Neuroradiol 2016;37:28-36 CrossRef Medline

14. Cohen J. A coefficient of agreement for nominal scales. Educ Psychol Meas 1960;20:37-46 CrossRef

15. Cohen J. Weighted kappa: nominal scale agreement with provision for scaled disagreement or partial credit. Psychol Bull 1968;70:21320 CrossRef Medline

16. Landis JR, Koch GG. The measurement of observer agreement for categorical data. Biometrics 1977;33:159-74 CrossRef

17. Bobek-Billewicz B, Stasik-Pres G, Majchrzak H, et al. Differentiation between brain tumor recurrence and radiation injury using perfusion, diffusion-weighted imaging and MR spectroscopy. Folia Neuropathol 2010;48:81-92 Medline

18. Wang S, Chen Y, Lal B, et al. Evaluation of radiation necrosis and malignant glioma in rat models using diffusion tensor MR imaging. J Neurooncol 2012;107:51-60 CrossRef Medline 